\title{
Sur les traces de Bamba Mos Xam : l'invention du théâtre mouride
}

\author{
Brian Valente-Quinn
}

\section{OpenEdition}

\section{Journals}

Édition électronique

URL : https://journals.openedition.org/coma/4233

DOI : $10.4000 /$ coma.4233

ISSN : 2275-1742

\section{Éditeur}

Institut des textes \& manuscrits modernes (ITEM)

\section{Référence électronique}

Brian Valente-Quinn, "Sur les traces de Bamba Mos Xam : l'invention du théâtre mouride », Continents manuscrits [En ligne], 13 | 2019, mis en ligne le 15 novembre 2019, consulté le 12 janvier 2023. URL http://journals.openedition.org/coma/4233 ; DOI : https://doi.org/10.4000/coma.4233

Ce document a été généré automatiquement le 12 janvier 2023.

\section{(c) $(5)$}

Creative Commons - Attribution - Pas d'Utilisation Commerciale - Pas de Modification 4.0 International - CC BY-NC-ND 4.0

https://creativecommons.org/licenses/by-nc-nd/4.0/ 


\title{
Sur les traces de Bamba Mos Xam : l'invention du théâtre mouride
}

\author{
Brian Valente-Quinn
}

1 Ce travail vise à mettre en lumière l'entrecroisement de deux champs d'études qui se rencontrent rarement dans la critique. La forte production culturelle du Sénégal dans les années qui suivent les Indépendances, ainsi que la riche iconographie visuelle de la confrérie mouride dans ce même pays, ont fait l'objet de nombreux travaux de recherche dans des domaines variés ${ }^{1}$. Les initiatives dans les arts de la scène du premier président sénégalais Léopold Sédar Senghor, notamment l'inauguration du Théâtre national Daniel Sorano avec sa troupe théâtrale de renommée internationale, et l'expansion du mouvement fondé par le cheikh soufi Amadou Bamba, semblent constituer deux approches distinctes de la culture nationale sénégalaise. L'une se caractérise par la défense d'une culture cosmopolite d'ouverture et de dialogue, l'autre par une soumission à l'enseignement d'un maître religieux dont la trajectoire spirituelle a profondément marqué l'imaginaire de la nation. Pourtant ces deux visions s'entrecroiseront à travers un spectacle ambulant d'inspiration religieuse intitulé Bamba Mos Xam, spectacle qui inaugure une nouvelle approche de la scène liant la pratique théâtrale aux préceptes de la croyance soufie si répandue dans ce pays.

2 Cet article rend compte d'une tentative de reconstitution mémorielle d'un spectacle évoqué à plusieurs reprises au cours d'une recherche sur le théâtre populaire sénégalais. Les acteurs clés rencontrés citaient la création scénique de langue wolof Bamba Mos Xam comme une réussite singulière pour son adaptation de la scène «à l'occidentale » aux attentes et aux désirs du grand public sénégalais ${ }^{2}$. Dans une initiative collaborative avec Mouhamadou Diol, directeur artistique de la compagnie théâtrale Kàddu Yaraax ${ }^{3}$, nous avons suivi les échos de cette création en cherchant, dans un premier temps, à apprécier la portée de sa tournée nationale dans les années 1970 et 80 . Nos premières discussions indiquaient d'emblée le souvenir durable de cette pièce, car ses anciens spectateurs - quasiment toute personne ayant la quarantaine ou plus - racontaient, sur des tons nostalgiques, l'effet de la pièce sur la population des quartiers qui se pressait pour la voir. Lors d'une conférence du festival 
de théâtre FEST'RAIL à Thiès, notre demande de renseignements auprès d'une cinquantaine d'acteurs culturels présents dans la salle, a suscité des réponses attestant de la mémoire populaire de cette pièce. L'artiste peintre Baba Ndiaye, membre influent de la culture thiessoise, est intervenu en rappelant à l'audience, qui acquiesçait à son propos, l'impact considérable de Bamba Mos Xam sur le public national de l'époque, précisant, « mais on a vu les gens entrer en transe en regardant cette pièce-là ${ }^{4}$ !"

3 Si la popularité de la création se présentait comme une évidence lors de nos recherches préliminaires, les détails de son histoire, son contenu, ses choix artistiques semblaient s'être quelque peu estompés avec le temps. En effet, malgré l'empreinte considérable de Bamba Mos Xam, ce spectacle n'a fait, jusqu'ici, l'objet d'aucun travail de recherche. Ceci est dû autant aux limites de nos disciplines académiques, dont les points aveugles et méthodes de recherche tendent à laisser en marge les formes de création ne correspondant pas aux usages conventionnels de la scène, qu'à la relation complexe qu'entretient l'institution mouride avec l'histoire de cette création non orthodoxe. C'est cette lacune de mémoire que nous avons cherché à remplir, d'une part pour examiner les innovations scéniques indispensables à l'incarnation d'une iconographie soufie; d'autre part pour tenter de valoriser l'apport de ce spectacle singulier au patrimoine culturel et religieux du pays.

4 En mobilisant nos contacts collectifs dans les milieux culturels et mourides des villes de Dakar, Touba, Thiès, Saint-Louis, Kaolack et Pikine, nous sommes partis à la recherche d'interlocuteurs pouvant nous informer sur l'histoire, les conditions et le contenu de la pièce, notamment ceux qui avaient participé à la tournée ou faisaient office d'acteurs culturels dans le milieu artistique de Kaolack, ville d'origine de la troupe; ou fréquentaient suffisamment le milieu de la muridiyya pour nous informer sur la réception du spectacle auprès de cette confrérie et de ses décideurs. Nous avons fini par échanger longuement avec neuf interlocuteurs principaux ${ }^{5}$ au cours d'une enquête qui nous a également permis de solliciter les souvenirs d'anciens spectateurs de la pièce. L'enquête a donné lieu à une collaboration fructueuse avec l'ancien directeur de la troupe ainsi qu'à une retranscription approximative (car citée de mémoire) de la pièce qui auparavant n'avait jamais été mise par écrit. Ce projet de recherche a également donné lieu à un court film documentaire collaboratif, dont l'objectif était de transmettre l'histoire de la création Bamba Mos Xam et d'examiner les enjeux de l'espace scénique qu'elle a créét.

\section{Entre fait social et objet esthétique}

5 Le domaine des études théâtrales, malgré la place importante qu'il accorde à la représentation, dépend souvent, dans ses analyses, des traces écrites de la création. Même dans les voies ouvertes par l'application de la critique génétique aux arts de la scène, l'approche du chercheur se définit par une lecture dynamique du « texte théâtral dans toutes ses composantes", avec le jeu scénique d'un côté et, de l'autre, la trace écrite du spectacle et de ses multiples processus de création ${ }^{7}$. Dans un contexte historique et culturel où l'écriture prime comme moyen de conservation et d'archivage cette méthodologie est certes porteuse. Toutefois, elle ne permet pas d'accéder à de très nombreuses formes d'expression dans des langues minoritaires dites "du Sud", car celles-ci ne laissent souvent pas de trace écrite. L'intégration des "expériences significatives de "terrain", librement inspirées de la démarche anthropologique ${ }^{8}$ ", 
peut, comme le précise Florent Siaud, fournir au chercheur un moyen de compléter l'interprétation des traces écrites, ou de compenser leur manque. Pourtant ce complément ne saurait à lui seul réhabiliter la mémoire d'une création passée. Dans son étude pluridisciplinaire sur la forme ghanéenne du concert party, Catherine Cole souligne le nombre insuffisant d'études prenant pour objet l'histoire et l'évolution des formes populaires en Afrique ou les innovations scéniques portées par les créateurs au cours du temps 9 . En effet, dans les études historiques des arts de la scène en Afrique, les méthodes de terrain et les approches purement textuelles présentent des limites. Le recours à des méthodologies hétérogènes, propres aux disciplines de l'histoire, de l'anthropologie, des études littéraires, voire de la pratique théâtrale, lorsque la recherche se base sur un processus d'observation participante, s'avère nécessaire ${ }^{10}$. Par ce bricolage, le chercheur vise à contourner l'indisponibilité des archives, et finit souvent par en créer une faite de matériau alternatif recueilli sur le terrain.

Cette recherche s'inscrit dans cette même logique de pluridisciplinarité mais prend surtout pour objet les innovations esthétiques de la création Bamba Mos Xam plutôt que son impact en tant que fait social. Elle rejoint les voix qui affirment un manque de contextualité dans les travaux sur le théâtre d'Afrique francophone, qui tendent à se focaliser sur les auteurs africains bénéficiant d'une présence artistique en Europe, mais n'ont finalement que peu exploré la vie de ces mêmes textes, ou la pratique théâtrale de manière générale, dans les contextes africains ${ }^{11}$. Les études sur le théâtre sénégalais témoignent d'un certain clivage entre l'approche esthétique et l'approche anthropologique. Souvent perçu comme d'inspiration traditionnelle et relevant d'une approche collective plutôt qu'auctoriale, le théâtre populaire devient l'apanage du terrain et des anthropologues. Mais ces approches populaires de la scène, dont on soulignera le lien aux cultures "orales", n'en aboutissent pas moins à des créations théâtrales dotées d'une forte volonté de recherche artistique et d'expérimentation esthétique. Elles peuvent être animées par un dialogue implicite avec des textualités méconnues par la critique théâtrale ou littéraire mais intégrées dans l'imaginaire collectif des publics populaires. De plus, elles laissent parfois des traces non écrites, qui échappent à toute tentative d'archivage, mais qui restent néanmoins lisibles dans les pratiques et les espaces scéniques investis par les générations suivantes. Elles ont trait au domaine mémoriel corporel et éphémère du «répertoire", pour employer ce concept porteur de Diana Taylor, plutôt qu'à celui des archives et de la mémoire écrite ${ }^{12}$.

\section{La création d'un espace scénique mouride}

7 Les débuts de Bamba Mos Xam remontent à la fin des années 1960 et notre premier défi consistait à trouver des acteurs ayant fait partie du premier noyau de la troupe. Nous avons vite été informés du décès du concepteur et metteur en scène du spectacle, Tamsir Ndiaye, et nos interlocuteurs nous orientaient donc vers Cheikh Fall, directeur de la troupe et responsable de sa logistique, que nous avons pu rencontrer sur son lieu de travail à l'Hôpital de Kaolack. À l'issue de cette rencontre, Fall nous a accordé deux journées entières de discussion, l'une pour parler de l'histoire de la création et de sa longue tournée, et l'autre pour nous assister dans la rédaction d'une retranscription de mémoire du contenu de la pièce ${ }^{13}$. 
8 Selon Fall, l'expérience de Bamba Mos Xam est née peu de temps après la participation non officielle de la troupe, qui s'appelait alors « L'Amicale Sérère Colette Senghor ", au célèbre Festival Mondial des Arts Nègres en 1966. Le nom de la compagnie rappelait l'identité sérère historique de sa région en même temps qu'il rendait hommage indirectement, par son épouse française, au président Senghor, lui aussi d'origine sérère. Cette tactique était courante pour les compagnies à la recherche de financements auprès des nouvelles institutions culturelles du gouvernement; elle ne portera pas ses fruits dans le cas de l'Amicale. Lasse de se voir refuser les soutiens qui nécessitaient souvent d'étroites relations avec les nouveaux centres d'influence politique de la capitale, la troupe change de stratégie. Son metteur en scène, Tamsir Ndiaye, propose aux membres d'adapter pour la scène la vie et l'œuvre du cheikh soufi Amadou Bamba, fondateur de la confrérie des mourides. À partir du récit bien connu de l'avènement, de l'exil et du retour triomphant du cheikh, la troupe met en scène un spectacle mêlant théâtre, musique et danse pour raconter dans un but artistique et pédagogique les faits de la vie du saint.

Dans nos entretiens, Fall nous a raconté à plusieurs reprises la première représentation publique du spectacle, qui eut lieu devant la maison du khalife général Serigne Fallou Mbacké, première figure de la confrérie. C'est de lui que la troupe espérait obtenir l'indispensable ndigal, qui lui permettrait de partir en tournée pour exploiter sa création $^{14}$. Sans la bénédiction explicite de Serigne Fallou, le spectacle aurait risqué de susciter la colère des adeptes ne trouvant pas à leur goût ce mélange inédit de croyance mouride et de pratiques théâtrales venues d'Occident, souvent jugées frivoles, voire indécentes. Le spectacle terminé, le khalife Serigne Fallou Mbacké, ravi de ce qu'il avait vu, en demanda le titre. Quand la troupe lui répondit «Bamba », il ajouta " Mos Xam », baptisant ainsi la création «Bamba Mos Xam ». La phrase signifie en wolof "goûter Bamba, c'est le découvrir». Elle exprime non seulement l'idée d'une expérience immersive mais aussi celle de la transmission d'un savoir qui passe par les sens plutôt que par la cognition.

10 Ces débuts propices marqueront le commencement d'une tournée théâtrale qui finira par sillonner tout le pays pendant plus de quinze années. Selon Fall, à son apogée la troupe gérait deux équipes simultanément afin de pouvoir assurer deux représentations sur la même date. Les acheteurs venaient directement au bureau de la troupe, qui se trouvait à Kaolack, et réservaient leur date en payant une partie du cachet total de 250000 francs CFA, une somme considérable, d'après Fall, surtout avant la dévaluation de cette monnaie en 1994. Le budget permettait à l'équipe de tourner, munie de projecteurs, d'une régie son, d'un camion vestiaire et d'une vingtaine d'acteurs, hommes et femmes, préparés à jouer plusieurs rôles différents en fonction des besoins de la troupe ${ }^{15}$. Ils se produisaient dans toutes les régions du pays, des plus grandes villes jusqu'aux villages les plus reculés. De Dakar à Tambacounda, en allant jusqu'en Casamance avec une courte tournée internationale qui les envoyait dans plusieurs pays de la sous-région, les comédiens de Bamba Mos Xam finirent par s'insérer dans l'imaginaire collectif, national et religieux du Sénégal et bénéficiaient d'une telle renommée qu'à la nouvelle de leur arrivée, les spectateurs affluaient, nombre d'entre eux se hissant même sur les toits des immeubles pour regarder de loin faute de pouvoir obtenir une place payante ${ }^{16}$. Selon Fall et les spectateurs consultés, le spectacle se jouait tous les jours et faisait toujours salle comble. 


\section{Une scène syncrétique entre art théâtral et immersion religieuse}

11 L'expérience de Bamba Mos Xam ne se caractérise pas uniquement par son succès financier et populaire; elle a également constitué une innovation radicale en ce qui concerne les usages possibles de l'espace scénique au Sénégal. Il en a résulté un nouvel espace théâtral que l'on peut qualifier de "syncrétique ", pour reprendre le terme employé par Christopher Balme qui décrit la stratégie, en contexte postcolonial, d'enrichir la scène occidentale de pratiques, de rites et de croyances locaux susceptibles de produire une certaine "décolonisation» de la pratique théâtrale ${ }^{17}$. En s'appropriant la scène théâtrale, historiquement transmise dans l'ancienne Afrique Occidentale Française par les écoles et les centres coloniaux ${ }^{18}$, pour mettre en corps et en espace la vie d'Amadou Bamba, cette création a ouvert la voie à une nouvelle vision du jeu scénique. Elle apparaît comme syncrétique dans la mesure où elle a laissé le champ libre à toute une dynamique d'inspiration soufie quant à l'acte d'incarner le saint fondateur et de raconter son histoire. En même temps, elle a préservé la possibilité d'une interaction non religieuse de la part d'un public non mouride, qui voyait dans la pièce la valorisation d'une figure nationale dans sa victoire contre les forces coloniales. Ainsi, le geste théâtral syncrétisé prenait une valeur à la fois sacrée et profane selon la réception de chaque spectateur et de chaque communauté, ce qui a permis au spectacle de résonner simultanément sur plusieurs registres et créé pour la compagnie une ambiguïté utile dont elle s'est servie tout au long de la tournée.

Il faut souligner que l'image d'Amadou Bamba est omniprésente au Sénégal et constitue la base d'une riche iconographie religieuse ${ }^{19}$. Elle ne sert pas uniquement de représentation neutre du saint, appelé par les adeptes Serigne Touba, ou le cheikh de Touba, la capitale religieuse de la confrérie. Elle rend présent le Serigne et sert à transmettre activement et par voie oculaire sa baraka, son aura ou sa bénédiction. Ainsi, une image de Serigne Touba ne peut faire simple office de métaphore visuelle car elle contient et transmet l'essence même du saint. Peut-elle donc être reproduite non pas par un rendu visuel comme c'en est la coutume, mais par le biais du corps d'un acteur? Les mourides doivent-ils avoir recours au même procédé de transmission de baraka dans la représentation théâtrale du saint ? C'est par les contours de leur création syncrétique que la troupe tente de répondre à ces questions dans le souhait de satisfaire à la fois son public populaire et les normes établies par les traditions mourides.

13 Par rapport aux autorités religieuses, il était de la plus grande importance que la troupe se fonde dans son adaptation sur les faits bien établis de l'hagiographie mouride ${ }^{20}$. C'est pourquoi, elle s'est tournée vers une tradition littéraire locale, écrite en wolofal, méconnue hors du pays mais fondamentale pour le "récit-maître » de la spiritualité mouride $^{21}$. Le wolofal est une forme de littérature ajami, qui transcrit la langue wolof en caractères arabes. L'un de ses plus grands poètes, Moussa Kâ, est l'auteur d'un long poème sur la vie d'Amadou Bamba qui se divise en deux parties : Jasaa u Sakóor u géej gi (Les Dons du Digne de Reconnaissance : Le Cycle de l'Océan) qui raconte l'exil de sept ans de Bamba au Gabon, et ensuite Yoonu jéeri ji (Le Cycle du continent), histoire de son exil de quatre ans en Mauritanie ${ }^{22}$. Kâ, un disciple d'Amadou Bamba, s'était engagé à écrire en wolofal, à la place de l'arabe qu'employait son maître, afin d'atteindre un public aussi large que possible. Sa version des faits de la vie de Bamba devient celle 
célébrée par des millions de mourides aujourd'hui. Elle raconte la persécution de Bamba par les colons français qui le soupçonnent, à tort, de vouloir faire la guerre dans la région contre l'expansion coloniale et célèbre, comme apothéose de la mission de Bamba, son départ en exil.

Le long poème de Kâ a longtemps servi d'outil pédagogique dans la propagation de la culture mouride au pays ${ }^{23}$. Dans le contexte post-Indépendance avant l'arrivée de la télévision au Sénégal, alors que les textes de Moussa Kâ n'étaient accessibles qu'à une élite encore capable de lire et de réciter la littérature wolofal, le théâtre est apparu aux décideurs mourides comme un outil pédagogique excellent qui venait remplacer le rôle tenu auparavant par la littérature ajami ${ }^{24}$. L'entreprise s'est avérée si productive pour la confrérie qu'elle envoyait de temps à autre des représentants qui voyageaient avec la troupe pour s'associer au spectacle et interagir avec les adeptes ou orienter les nonadeptes vers l'initiation (par la soumission au cheikh, ou le jébalu en wolof) ${ }^{25}$.

Pourtant le succès du spectacle ne dépendait pas uniquement de son aspect religieux, car il jouait aussi sur le positionnement ambigu d'une visée à la fois sacrée et profane. Cette ambiguïté concernait également l'identité religieuse des comédiens, qui n'appartenaient pas tous à la confrérie mouride. D'une part, il aurait été impensable que Bamba soit interprété par un comédien qui n'était pas lui-même un adepte, ou talibé, du cheikh. En effet, l'acteur vedette de la troupe, Demba Ndiaye, intégrait jusque dans sa vie personnelle et spirituelle la lourde responsabilité de jouer le rôle de Bamba en prenant soin d'imiter la voix, la démarche, la physionomie du saint, se consacrant également à l'étude dévouée et scrupuleuse des écrits et de la vie de $\mathrm{Bamba}^{26}$. D'autre part, Cheikh Fall a longuement insisté durant nos entretiens sur la composition hétérogène de la troupe : le metteur en scène à l'origine du concept, Tamsir Ndiaye, appartenait, lui, à la confrérie tidjane et la troupe, selon Fall, comprenait même des chrétiens. Ce détail importe à deux égards. D'abord il souligne que, malgré l'effet de dissémination religieuse qui a, en quelque sorte, porté le succès du spectacle, la création ne représentait pas pour autant un pur projet de prosélytisme. Bien au contraire, il maintenait au sein de son effectif aussi bien que dans son contenu et ses publics ciblés, une vision foncièrement inclusive des différentes appartenances religieuses et de leur place dans le récit de Bamba. Parmi les figures centrales de l'histoire mouride de cette époque, la pièce mettait en valeur la contribution d'autres pionniers et résistants des confréries majeures du Sénégal. Ainsi, la pièce débutait, selon sa retranscription, avec l'entrée de El Hadj Cheikh Oumar Tall, résistant au colonialisme et héros de la confrérie tidjane, qui prédit, dans un dialogue avec les futurs parents de Bamba, l'arrivée imminente au sein de leur famille d'un très grand marabout ${ }^{27}$. De tels échanges sur scène permettaient aux spectateurs non mourides de s'impliquer dans le déroulement du spectacle aux côtés de leurs compatriotes mourides. Ils minimisaient, de plus, toute éventuelle concurrence entre les appartenances religieuses, reflétant ainsi les pratiques de tolérance caractéristiques de ce que l'on a pu nommer « l'exception sénégalaise ${ }^{28}$ ».

16 L'insistance sur la nature hétérogène de la troupe dévoile, de surcroît, une revendication chez Cheikh Fall d'assumer pleinement l'aspect théâtral et artistique du spectacle Bamba Mos Xam qui, bien qu'il applique une approche de la représentation et de la corporalité sur scène que l'on pourrait qualifier de mystique, n'en reste pas moins une création jouée par des acteurs capables d'incarner un rôle et d'en sortir. L'ambiguïté de ce nouvel espace scénique a nécessité, selon Fall, une longue période 
d'apprentissage pour un public populaire qui n'acceptait pas toujours la distance ontologique présumée entre l'effigie de Bamba et l'acteur qui l'incarnait sur scène. Surtout aux débuts de leur tournée, les comédiens ne savaient jamais d'un moment à l'autre si un membre du public allait prendre l'initiative de monter sur scène pour intervenir physiquement dans l'histoire qui était en train de se jouer ${ }^{29}$. Si l'on accepte la notion qu'interpréter Bamba constitue bien plus qu'un simple jeu de rôle mais une transmission de son essence, il devient inacceptable, pour un adepte, de voir la figure du saint menacée ou malmenée par les représentants du colonialisme. Le procès de Bamba dans la pièce en présence du gouverneur général à Saint-Louis (nommé Borom Ndar dans le poème de Kâ) présentait une situation particulièrement sensible ${ }^{30}$. L'acteur qui incarnait le Borom Ndar se voyait régulièrement agressé par des spectateurs qui surgissaient du public pour corriger le colon français pour son arrogance à l'égard de Bamba. Dans les distributions, il fallait prendre ce danger en compte et donner les rôles des adversaires français et africains du cheikh à des acteurs suffisamment grands pour parer une éventuelle agression venant du public.

Ces difficultés mises à part, la troupe a néanmoins su tirer profit, artistiquement et financièrement, des particularités de cet espace de jeu en faisant preuve d'une fine compréhension des croyances et des comportements religieux des mourides. À chaque représentation un certain nombre d'adeptes avançait subitement vers la scène au milieu du spectacle pour faire un don ostentatoire d'une poignée de billets, un geste qui exprimait à la fois leur plaisir de voir et d'entendre les louanges de leur maître et leur dévouement au cheikh. De là est survenu spontanément et au cours des représentations un rituel régulier du don de la part des spectateurs les plus fervents qui a fini par s'associer à la mémoire de la théâtralité même du spectacle. Ce geste correspondait pour les croyants à la pratique bien établie de la hadiyya, une obligation par laquelle l'aspirant (le sens du mot "mouride ») exprime sa gratitude envers son cheikh en lui faisant un don qui sert aussi à opérer un échange d'amour et de baraka $a^{31}$. Sur scène, le maintien d'une pratique similaire plaçait le spectacle encore plus dans un univers mouride d'échange par le biais d'une relation interactive avec le public qui contribuait bien évidemment au succès de la troupe.

L'interactivité que facilitait l'espace scénique de Bamba Mos Xam ne se limitait pas à des échanges financiers car on pouvait aussi prendre part à l'énergie de l'événement par la transe spirituelle. Les représentations étaient régulièrement interrompues par des spectateurs qui émettaient soudainement des cris avant de s'évanouir, en gesticulant de manière désordonnée. Il appartenait ensuite aux autres spectateurs de retenir physiquement la personne pour qu'elle ne se fasse pas mal. La manifestation de la transe dans le cas des spectateurs de Bamba Mos Xam indiquait que l'individu était possédé par la baraka de Bamba, dont l'essence ne manquait pas d'émouvoir à l'excès les plus fervents dévoués, qui pouvaient même perdre le contrôle de leur propre corps tellement la force spirituelle de Bamba les dépassait. Ainsi pour tout spectateur dans cet espace syncrétique, l'accord implicite qui régit la manière dont il doit interagir avec le spectacle change radicalement lorsqu'il devient clair qu'il ne s'agit nullement de regarder passivement une simple histoire. Pour les adeptes, la transmission oculaire de la baraka de Bamba avait bel et bien lieu, renforcée d'autant plus par le jeu de l'acteur et par la nature immersive du spectacle. Ceci ajoutait à l'expérience une certaine imprévisibilité, presqu'un danger palpitant, dont l'effet, tout aussi théâtral que spirituel, était de se demander à tout moment si un voisin pouvait inopinément se faire 
posséder par la baraka de Bamba, voire si l'on était soi-même susceptible d'entrer en transe.

\section{Les traces hétérogènes de la création}

19 Si l'on sait que Bamba Mos Xam a clôturé sa tournée dans les années 1980 (l'année exacte n'a pas pu être déterminée), nos interlocuteurs en ignorent les raisons précises. Le mystère qui entoure rétrospectivement sa fin abrupte a nourri un certain nombre de rumeurs et de théories. Certaines se fondent sur la pratique inhabituelle d'incarner Bamba, geste qui impliquerait une charge spirituelle trop lourde pour le commun des mortels, et le décès en tournée de l'acteur principal Demba Ndiaye a sûrement dû renforcer aux yeux de certains la crédibilité de cette éventualité. D'autres se focalisent sur les sommes considérables récoltées par la troupe qui auraient fini soit par diviser le groupe soit par déplaire aux autorités mourides jugeant inapproprié l'acte de s'enrichir personnellement par le récit fondateur de la confrérie. Cheikh Fall a tenté d'y apporter des clarifications lors de nos discussions en dénonçant ces théories. Il précise que les acteurs de la troupe avaient tout simplement vieilli sans pouvoir transmettre le spectacle à une jeune génération d'artistes, car toute tentative de passer le relais se serait compliqué, dit-il, par le fait que l'autorisation de jouer ne leur accordait pas pour autant la permission de transmettre.

Nonobstant les obstacles à la transmission élucidés par Fall, on peut également constater un silence institutionnel autour de ce spectacle sûrement dû à sa position dans les interstices de l'art théâtral et de l'immersion religieuse. Son histoire et son exemple semblent ne pas avoir été revendiqués durablement par les institutions mourides et, malgré le succès retentissant de Bamba Mos Xam, la tournée reste une expérience unique dans son genre au Sénégal. Si nous n'avons pu établir la position officielle de la muridiyya, ni celle de l'époque, ni celle d'aujourd'hui, par rapport à la création, sa fin abrupte pourrait laisser entendre que le soutien des décideurs mourides n'était pas unanime. Le manque de mémoire institutionnelle concerne également le Théâtre national Daniel Sorano, scène mythique de l'époque et lieu de création où la troupe s'est pourtant produite à plusieurs reprises. Les archives du théâtre ne contiennent aucune trace de ces représentations ${ }^{32}$. Il est également à noter que, dans ses volumineux écrits sur la production culturelle sénégalaise, Léopold Sédar Senghor ne fait aucune mention de ce succès théâtral inouï qu'il ne pouvait ignorer. En effet, ce théâtre mouride ressemblait bien plus au théâtre populaire sénégalais qu'au théâtre d'écrivains que prônait le poète-président, qui voyait dans les arts populaires un phénomène secondaire par rapport à la plus haute culture nationale qu'il souhaitait promouvoir ${ }^{33}$.

21 L'espace théâtral créé par Bamba Mos Xam a néanmoins pu contribuer à poser des jalons pour les multiples tentatives suivantes de mêler arts populaires ou urbains à un programme religieux prenant pour interlocuteur l'intégralité de la nation, autant dans les arts visuels que dans des formes musicales telles que le mbalax ou le rap ${ }^{34}$. En effet, l'expérience préfigure une certaine volonté, courante dans le Sénégal contemporain, de transmettre des exemples et des leçons puisés dans la vie et l'œuvre des figures religieuses iconiques du pays. Si le manque de traces écrites de ce spectacle complique l'étude de son histoire, les quelques indications récoltées sur ses particularités et orientations scéniques peuvent nous permettre de discerner, sans croire à une 
continuité directe, les contours du terrain artistique qu'il a su baliser pour les artistes des générations suivantes.

C'est dans l'objectif d'examiner les manifestations contemporaines d'un tel espace théâtral syncrétique que nous avons participé à un projet de transmission à l'initiative de Diol et de la jeune compagnie théâtrale Sant Yàlla de Yeumbeul. Dans ce cadre nous avons organisé une rencontre entre Cheikh Fall et la troupe (dont le nom signifie « merci à Dieu »), qui a créé un spectacle pluridisciplinaire du titre de « Jaar Jaari Mame Cheikh Ibrahima Fall », ou « La Vie de Mame Cheikh Ibrahima Fall », le premier disciple d'Amadou Bamba. Cette pièce tourne depuis sa création dans les milieux populaires des centres culturels et suscite des réactions faisant écho à l'expérience de Bamba Mos Xam. Très tôt dans son processus de création, la troupe, qui s'inspire de plusieurs formes traditionnelles, notamment africaines et italiennes suite à une longue collaboration avec une compagnie théâtrale à Ravenne, s'est vue comme la troupe précédente dans l'obligation de se déplacer à Touba pour demander le ndigal qui lui accorderait l'autorisation de se produire publiquement. Le metteur en scène, Adama Gueye, précise que les spectateurs de l'ancienne génération faisaient déjà la parallèle entre leur spectacle et celui de Bamba Mos Xam et, lors de leur voyage à Touba, on a qualifié leur création de «Bamba Mos Xam $2 »^{35}$. La remarque a poussé les jeunes comédiens à en savoir plus sur cette création dont ils ignoraient l'existence à travers un échange avec Cheikh Fall ${ }^{36}$.

Nous avons assisté à Yeumbeul à une représentation du spectacle de Sant Yàlla ${ }^{37}$, qui intègre danses et musiques, mélangeant des styles traditionnels et contemporains pour mobiliser par ses costumes et le comportement des acteurs une imagerie évoquant la figure du Baye Fall, ou celui qui se dévoue à l'imitation de l'exemple de Mame Cheikh Ibrahima Fall. Leur spectacle s'ouvre sur une longue introduction musicale menée par le chef percussionniste et suivie par un chœur d'hommes qui entonne un chant bien connu de la culture mouride. Les chants jouent un rôle important dans l'aspect performatif du Baye Fall, ce qui permet aux artistes de Sant Yàlla de faire valoir leurs talents de musiciens et de danseurs. Ce chant, familier aux non-adeptes comme aux adeptes, évoque la puissance spirituelle totale de Bamba : Yaa ñuy moom ou « c'est à toi (Bamba) que nous appartenons $»^{38}$. À l'entonnement de ces paroles, les réactions fusent de la part d'un groupe de jeunes spectateurs qui crient et se mettent à danser. L'un d'eux finira par entrer subitement en transe au milieu du spectacle qui s'arrête le temps de sécuriser la personne. Par la suite, nous assisterons également au rite du don monétaire lorsqu'un homme jaillit sur scène pour poser une liasse de billets, déclenchant ainsi un défilé de spectateurs qui suivent son exemple.

Si la possibilité d'une archive, ou trace écrite, de Bamba Mos Xam s'est déjà effacée avec le temps, il ne faut pas pour autant négliger l'empreinte de ce spectacle sur la mise en corps d'un certain mode d'engagement, à la fois culturel et dévotionnel, avec les limites, les conventions et les possibilités du jeu scénique. En effet, l'émergence d'une troupe comme Sant Yàlla reflète la possibilité d'une autre transmission que par la trace écrite, qui permettrait à un héritage longtemps oublié de revivre grâce à une réappropriation par une nouvelle génération. Au-delà des soucis de conservation du texte, les particularités du jeu scénique permettent une transmission par la mémoire $\mathrm{du}$ corps et des espaces scéniques ayant porté les multiples innovations de leurs créateurs. C'est cette caractéristique même du jeu théâtral qui nous permet de suivre les traces hétérogènes des créations qui sont passées dans l'histoire, mais toujours, en 
quelque sorte, présentes dans leurs contextes culturels africains, comme c'est ostensiblement le cas pour Bamba Mos Xam.

\section{BIBLIOGRAPHIE}

BABOU, Cheikh, Fighting the Great Jihad: Amadu Bamba and the Founding of the Muridiyya of Senegal, 1853-1913, Athens, Ohio University Press, 2007.

BALME, Christopher, Decolonizing the Stage: Theatrical Syncretism and Post-Colonial Drama, Oxford, Clarendon Press, 1999.

BENGA, Ndiouga, « Mise en scène de la culture et espace public au Sénégal », Afrique et Développement, vol. 35, $\mathrm{n}^{\circ}$ 4, 2010, 237-260.

CAMARA, Sana, «A'jami Literature in Senegal: The Example of Sëriñ Muusaa Ka, Poet and Biographer ", Research in African Literatures, vol. 28, n³, 1997, p. 163-182.

CAMARA, Sana, Sheikh Ahmadu Bamba: Selected Poems, Leiden, Brill, 2017.

CANELLA, Gino et Brian VALENTE-QUINN (réal.), Bamba, The Taste of Knowledge, film documentaire, 2018, 22 min.

COHEN, Joshua, « Locating Senghor's École de Dakar: International and Transnational Dimensions to Senegalese Modern Art, c. 1959-1980 », African Arts, vol. 51 n³, 2018, p. 10-25.

COLE, Catherine, Ghana's Concert Party Theatre, Bloomington, Indiana University Press, 2001. CONTEH-MORGAN, John, Theatre and Drama in Francophone Africa: A Critical Introduction, Cambridge, Cambridge University Press, 1994.

CRUISE O’BRIEN, Donald, « The Senegalese Exception », Africa, vol. 66, n 3, 1996, p. 458-64. DIAGNE, Souleymane Bachir, Léopold Sédar Senghor: L'Art africain comme philosophie, Paris, Riveneuve éditions, 2007.

DIENG, Bassirou, L'Épopée de Cheikh Amadou Bamba de Serigne Moussa Kâ, Dakar, Presses universitaires de Dakar, 2006.

DONKOR, David, Spiders of the Market: Ghanaian Trickster Performance in a Web of Neoliberalism, Bloomington, Indiana Unversity Press, 2016.

EDMONDSON, Laura, Performance and Politics in Tanzania: The Nation on Stage, Bloomington, Indiana University Press, 2007.

FÉRAL, Josette, «Introduction: Towards a Genetic Study of Performance - Take 2 », Theatre

Research International, vol. 33, $\mathrm{n}^{\circ}$ 3, 2008, p. 223-333.

HARNEY, Elizabeth, In Senghor's Shadow: Art, Politics, and the Avant-Garde in Senegal, 1960-1995, Durham, Duke University Press, 2004.

HILL, Joseph, « "Baay is the spiritual leader of the rappers": performing Islamic reasoning in Senegalese hip-hop ", Contemporary Islam, vol. 10, n² 2, 2016, p. 267-287.

JÉZÉQUEL, Jean-Hervé, « Le "théâtre des instituteurs” en AOF (1930-1950) : pratique socioculturelle et vecteur de cristallisation de nouvelles identités urbaines », dans Fêtes urbaines en Afrique : espaces, identités et pouvoirs, Odile GEORG (dir.), Paris, Karthala, 1994, p. 181-200. LE LAY, Maëline, La Parole construit le pays. Théâtre, langues et didactisme au Katanga (République Démocratique du Congo), Paris, Honoré Champion, 2014.

LÉGER, Nathalie et Almuth GRÉSILLON, "Scènes de genèses théâtrales ", Genesis, n 26, 2005, p. 19-34. MCLAUGHLIN, Fiona, «Islam and Popular Music in Senegal: The Emergence of a "New Tradition" », 
Africa: Journal of the International African Institute, vol. 67, n 4, 1997, p. 560-581.

MURPHY, David (dir.), The First World Festival of Negro Arts, Dakar, 1966, Liverpool, Liverpool University Press, 2016.

NEVEU-KRIGELBACK, Hélène, Dance Circles: Movement, Morality and Self-Fashioning in Urban Senegal, Oxford, Berghahn, 2013.

NGOM, Fallou, Muslims Beyond the Arab World: The Odyssey of Ajami and the Muridiyya, New York, Oxford University Press, 2016.

NIANG, ABDOULAYE, " "Preaching Music" and Islam in Senegal: Can the secular mediate the religious? The case of rap and mbalax music ", African Communication Research, vol. 2, $\mathrm{n}^{\circ}$ 1, 2009, p. 61-84.

ROBERTS, Allen F. et Mary Nooter ROBERTS, A Saint in the City: Sufi Arts of Urban Senegal, Los Angeles, UCLA Fowler Museum of Cultural History, 2003.

ROBERTS, Allen F., " Citoyennetés visuelles en compétition dans le Sénégal contemporain », Les Arts de la citoyenneté au Sénégal, Mamadou Diouf et Rosalind Fredericks (dir.), Paris, Karthala, 2013, p. 195-236.

SHIPLEY, Jesse Weaver, Trickster Theater: The Poetics of Freedom in Urban Africa, Bloomington, Indiana University Press, 2015.

SIAUD, Florent, « La génétique de la mise en scène à l'épreuve de l'expérience humaine », $L a$ Fabrique du texte à l'épreuve de la génétique, Olga ANOKHINA et Fatiha IDMHAND (dir.), Éditions des archives contemporaines, France, 2018, p. 29-43.

SNIPE, Tracey, Arts and Politics in Senegal, 1960-1996, Trenton NJ, Africa World Press, 1998.

TANG, Patricia, Masters of the Sabar: Wolof Griot Percussionists of Senegal, Philadephia, Temple University Press, 2007.

TAYLOR, Diana, The Archive and the Repertoire: Performing Cultural Memory in the Americas, Durham, Duke University Press, 2003.

\section{ANNEXES}

Fig. 1 : Image du spectacle Bamba Mos Xam : Bamba est jugé par le gouverneur général à Saint-Louis (photo de Cheikh Fall ; image tirée du film Bamba, The Taste of Knowledge). 


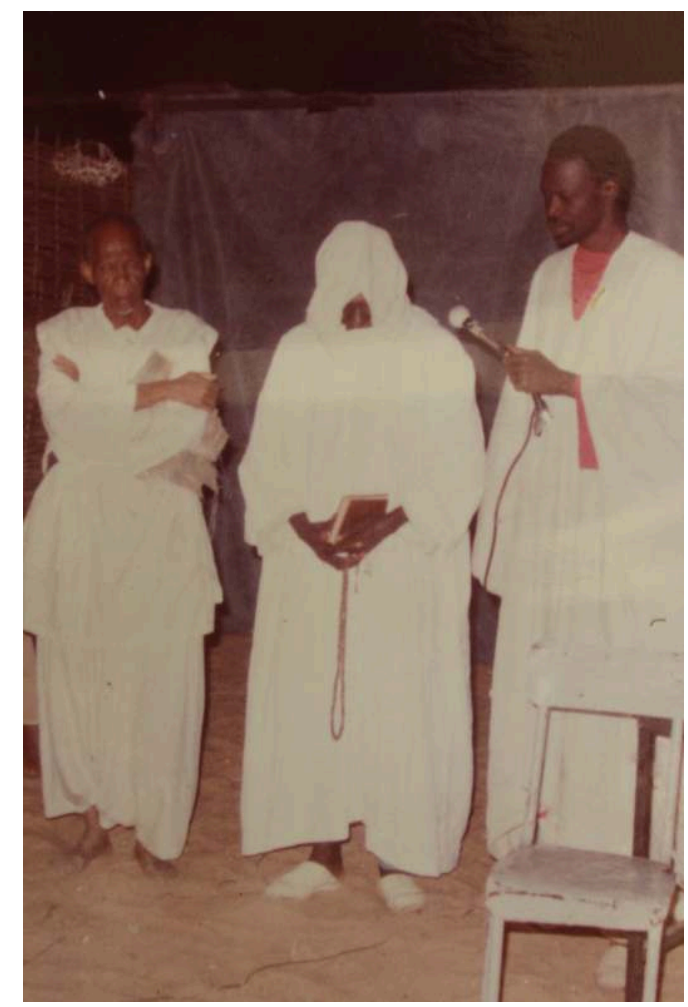

Fig. 2 : Un djinn tourmente Bamba lors de son exil au Gabon (photo de Cheikh Fall ; image tirée du film Bamba, The Taste of Knowledge).

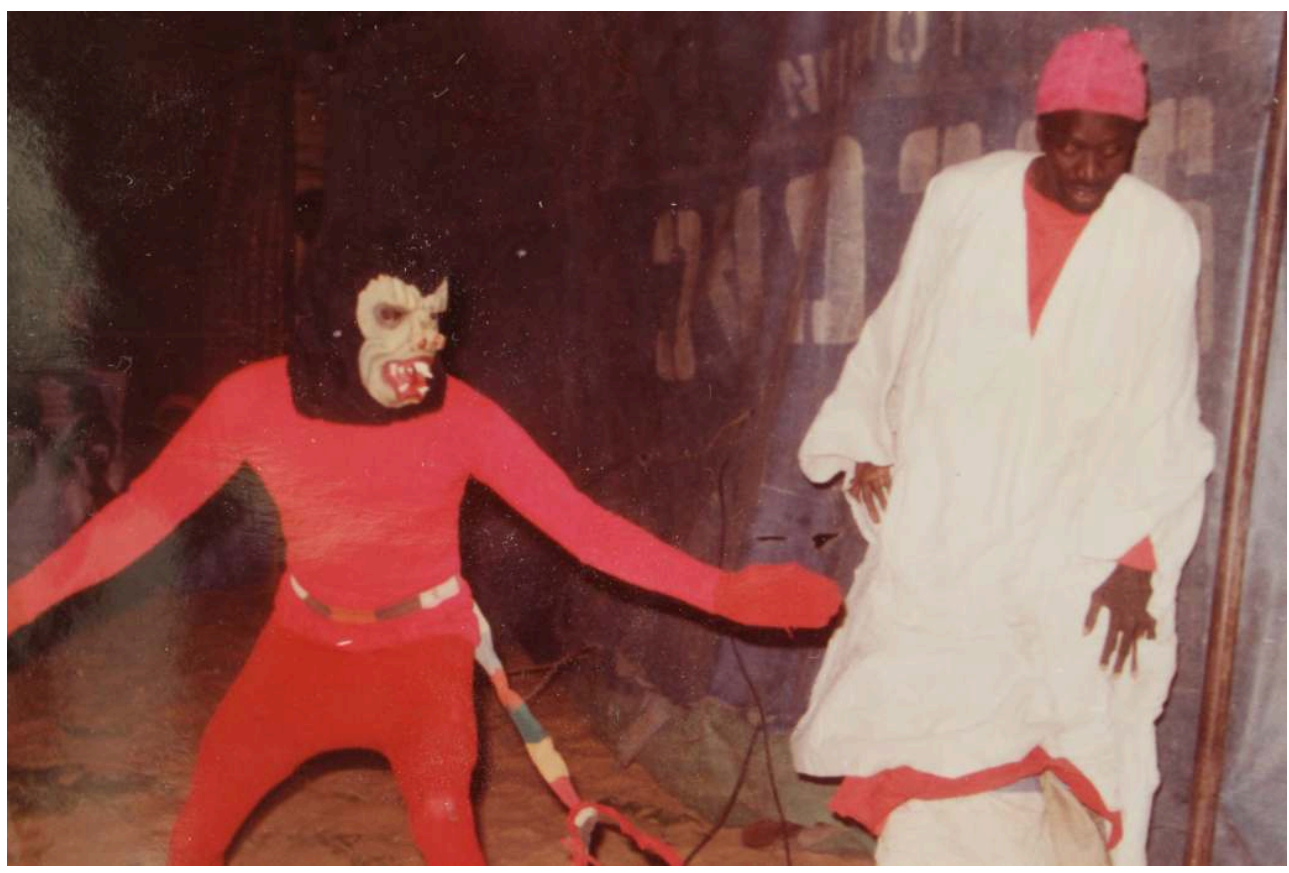

Fig. 3 : Cheikh Fall (au centre, en rouge) dans le rôle de Tanor Gogne, persécuteur des talibés de Bamba (photo de Cheikh Fall ; image tirée du film Bamba, The Taste of Knowledge). 


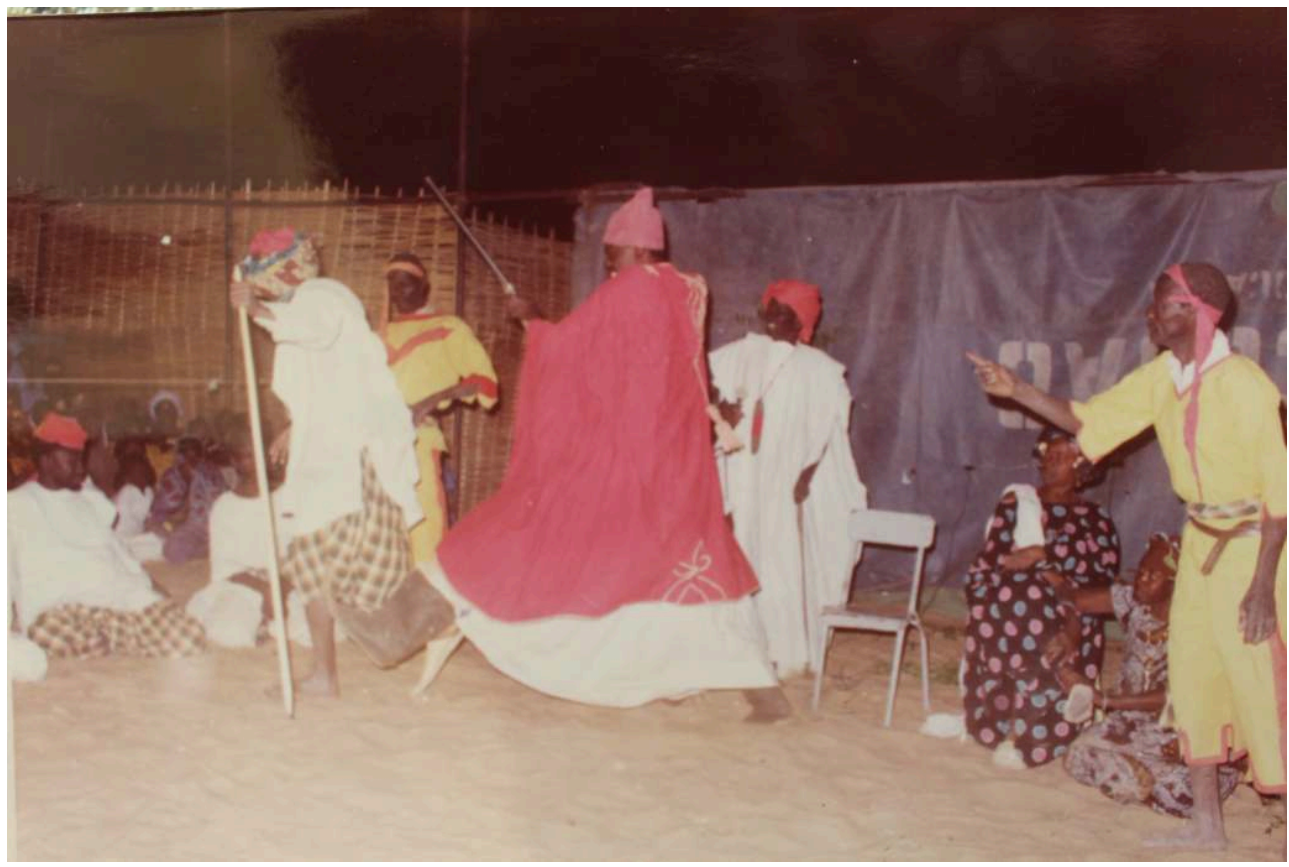

Fig. 4 : Rencontre entre Cheikh Fall de la troupe Bamba Mos Xam et les artistes de la compagnie Sant Yàlla (image tirée du film Bamba, The Taste of Knowledge).

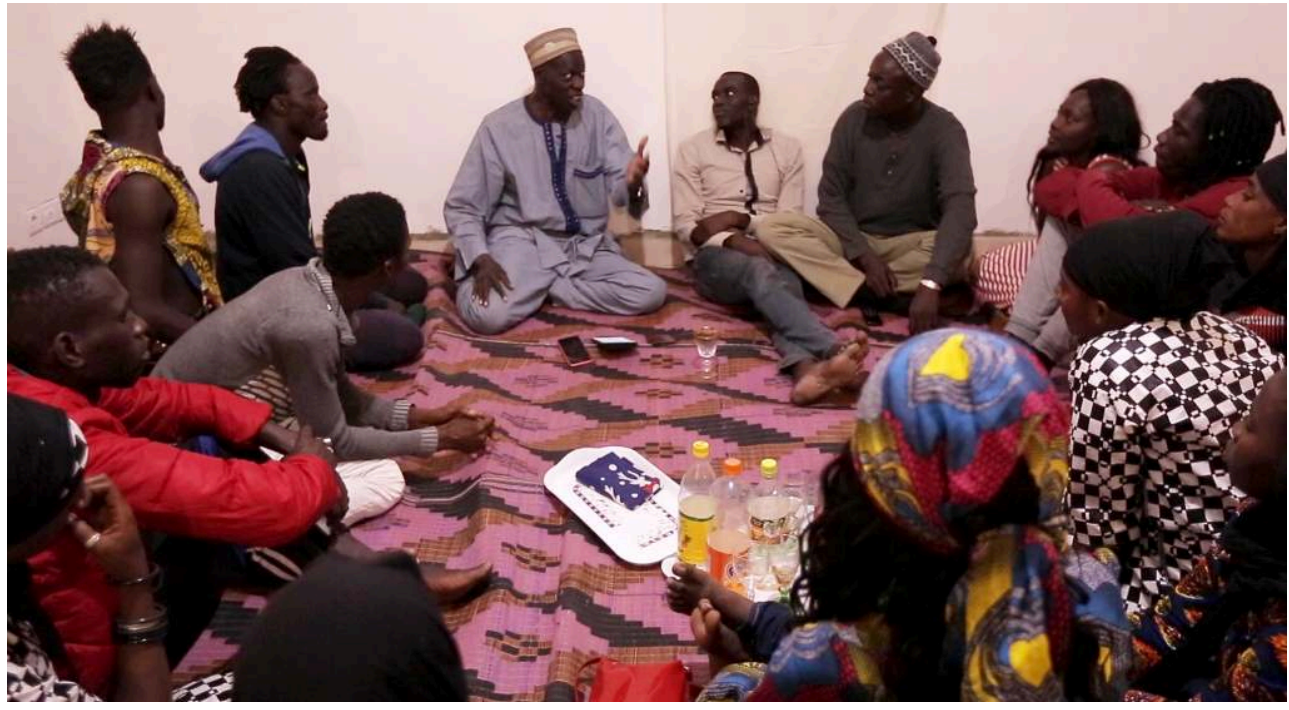

Fig. 5 : Xadim Bèye, acteur principal de La Compagnie Sant Yàlla, raconte et interprète les faits de la vie de Mame Cheikh Ibrahima Fall, chef des Baye Fall (image d'une répétition à Thiaroye). 


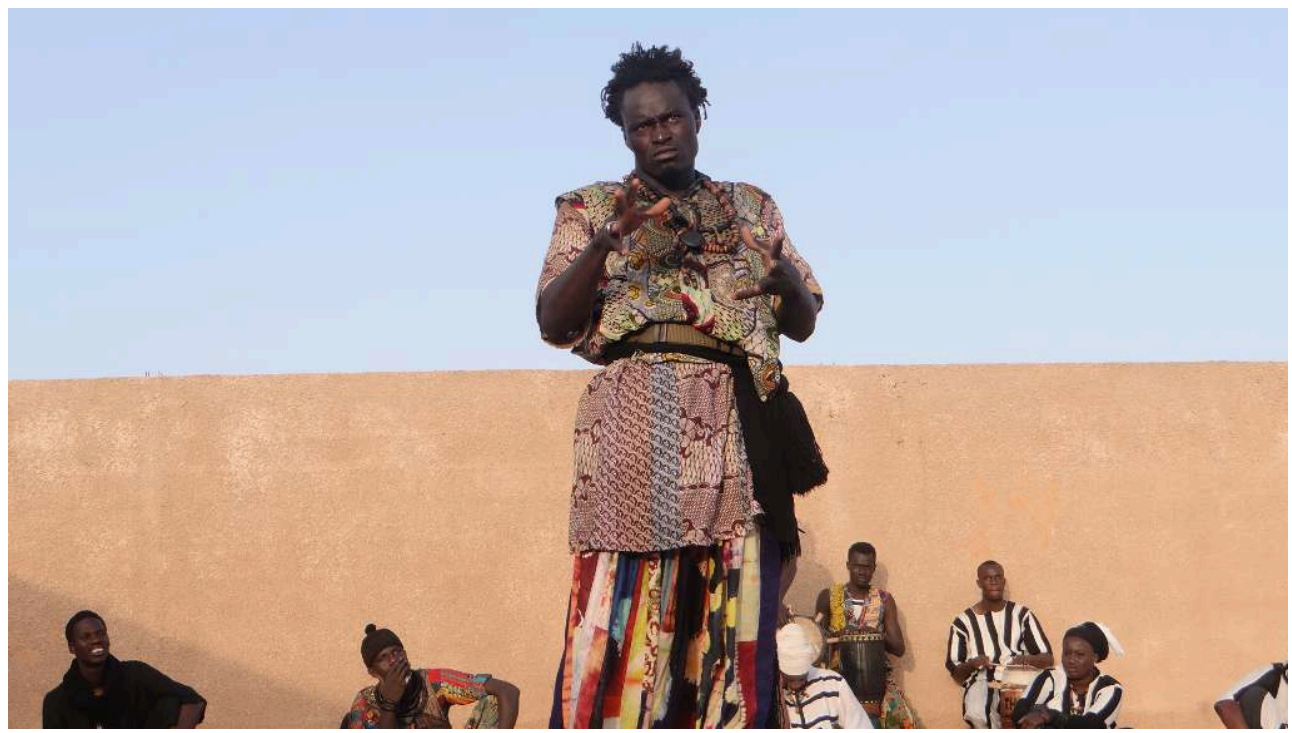

Fig. 6 : Le chef percussionniste Tapha Mbaye démarre le spectacle (image d'une répétition à Thiaroye).

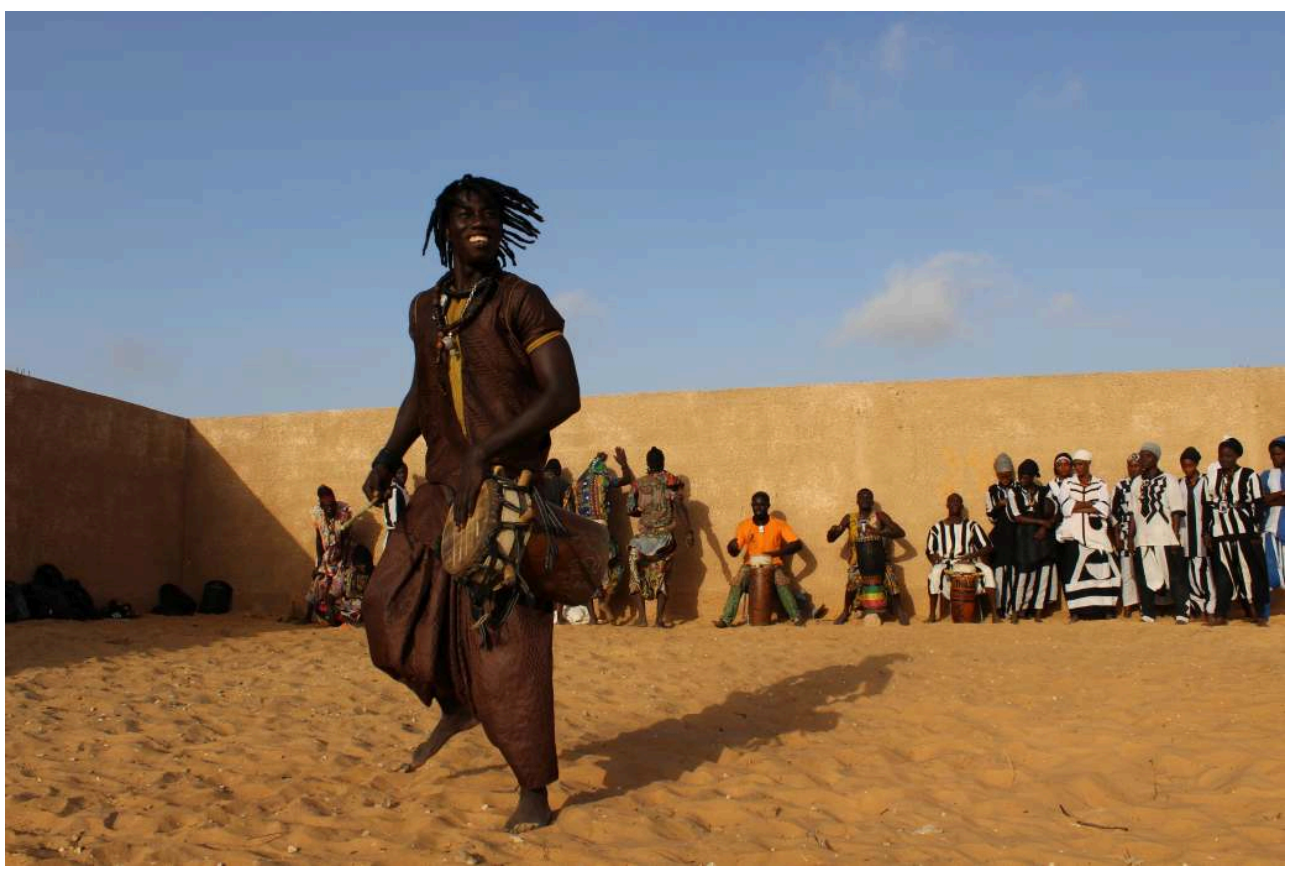

Extraits de Bamba Mos Xam (en Anglais, puis en français) 


\begin{tabular}{|c|c|}
\hline \multicolumn{2}{|c|}{ Excerpts from the opening sequence of scenes from Bamba Mos Xam } \\
\hline \multicolumn{2}{|c|}{$\begin{array}{l}\text { Act } 1 \text { - Shaykh Umar Fuutiyu Taal's voyage in the Rip }{ }^{1} \\
\text { Scene 1 - Encounter with Maba Jaxu Ba }\end{array}$} \\
\hline \multicolumn{2}{|c|}{$\begin{array}{l}\text { Along a road. } \\
\text { Al-Haij Shaykh Umar Taal and his young taalibe (disciple) enter, } \\
\text { accompanied by Maba Jaxu Ba. } \\
\text { They stop at a crossroads. }\end{array}$} \\
\hline Umar Taal: Maba Jaxu! & Umar Taal: Maba Jaxu! \\
\hline Maba Jaxu: Labbayka! & Maba Jaxu: Here I am! \\
\hline $\begin{array}{l}\text { Umar Taal: Yemal fii, fii baax na. Waaye boo } \\
\text { demee na nga góor-góorlu ci Islaam. }\end{array}$ & $\begin{array}{l}\text { Umar Taal: You may return I can carry on } \\
\text { from here. Continue working for the good of } \\
\text { Islam }\end{array}$ \\
\hline $\begin{array}{l}\text { Maba Jaxu: Tabaarkàlla! Waaye sëriñ bi Taal, } \\
\text { dama bëggoon nga may ma ndigal ma fétal } \\
\text { jihad. }\end{array}$ & $\begin{array}{l}\text { Maba Jaxu: Thanks to God! But, Sëriñ Taal, I } \\
\text { wished to ask for your permission to wage } \\
\text { holy war. }\end{array}$ \\
\hline Umar Taal: Fétal jihad ngir Islaam? & Umar Faal: Holy war for Islam? \\
\hline Maba Jaxu: Waaw. & Maba Jaxu: Yes. \\
\hline $\begin{array}{l}\text { Umar Taal: May nanu la ko. Waaye boo } \\
\text { demee ba bërëb booba ñuy wax Siin, na nga } \\
\text { têral sa ngànnaay. Laamissol ak ñoom. Sunuy } \\
\text { gàmmu lañu. Soo fa yékètee ngànnaay, gis } \\
\text { naa sa dugg Siin waaye gisuma sa génn Siin. } \\
\text { Tällalal say loxo. }\end{array}$ & $\begin{array}{l}\text { Umar Taal: It is granted. But when you arrive } \\
\text { in the place called Siin, lay down your arms. } \\
\text { Be conciliatory with them. They are our } \\
\text { brethren If you take up arms there, I see you } \\
\text { going into Sin, but not coming out. }{ }^{4} \text { Hold out } \\
\text { your hands. }\end{array}$ \\
\hline \multicolumn{2}{|c|}{ Maba Jaxu holds out his hands and they pray } \\
\hline $\begin{array}{l}\text { Umar Taal: Won ma yoonu Mbàkke Bawol. } \\
\text { Maba Jaxu: Da ngay jaar nii, boo demee ba ci } \\
\text { dëkk bu ñuy wax Poroxaan, soo demee, }\end{array}$ & $\begin{array}{l}\text { Umar Taal: Show me the way to Mbakke } \\
\text { Bawol. } \\
\text { Maba Jaxu: Take that road until you've } \\
\text { arrived at the town called Poroxaan. When }\end{array}$ \\
\hline
\end{tabular}

${ }^{1}$ Region that will become the Rip Kingdom under the rule of Maba Jaxu Ba, and whose capital, Nioro du Rip, is named after the city Nioro du Sahel, in Mali, conquered by Shaykh Umar Taal ${ }^{2}$ A clerical warrior who contributed to the spread of the Tijaani brotherhood in Senegal. ${ }_{3}^{3}$ A disciple of Umar Taal who later becomes the Almamy of the Saloum Kingdom. ${ }^{4}$ Here, Umar Taal predicts Maba Jaxu's defeat and death in 1868 against the forces of the

animist Serer population of Sin.
${ }^{3}$ The ancestral city of Amadu Bamba, in the region of Bawol.

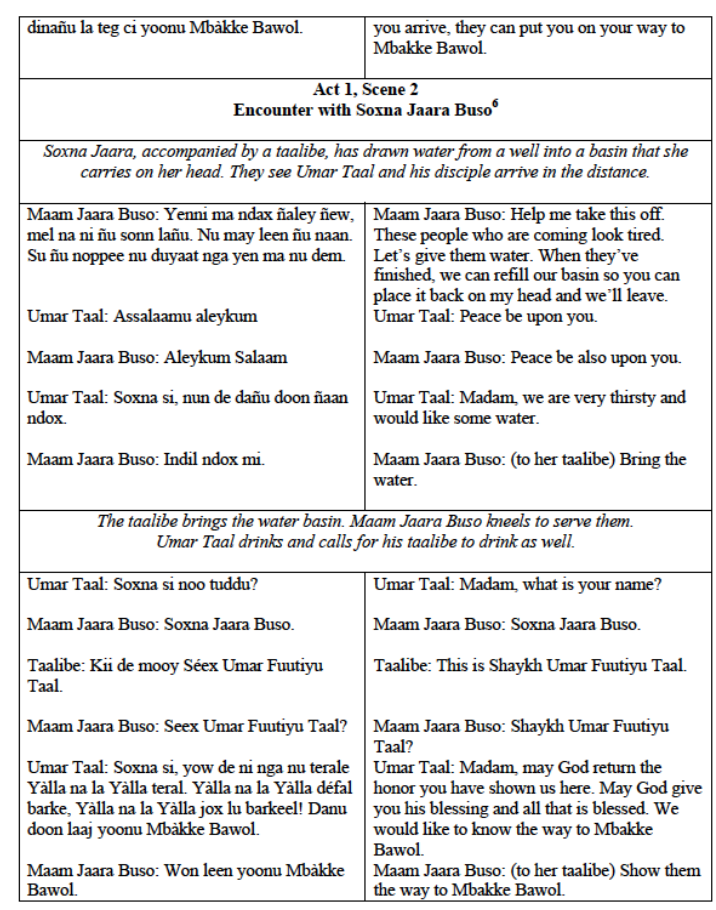

${ }^{6}$ The mother of Amadu Bamba. Her home city of Poroxaan is today a pilgrimage site for Murids. 


\begin{tabular}{|l|l|}
\hline \multicolumn{2}{|c|}{ Her taalibe shows them the way to Mbakke Bawol and they depart. } \\
\hline \multicolumn{2}{|c|}{ Act l, Scene 3 } \\
\hline \multicolumn{2}{|c|}{ Encounter with Maam Moor Anta Sali' } \\
They all have a seat in the city square.
\end{tabular}

${ }_{8}^{7}$ Amadu Bamba's father and a learned qadi, or scholar of Islamic law.

${ }^{8}$ Anta Sali was a devotee of the Qadiriyya, a Sufi order founded in Bagdad in the twelfth century and brought to Senegal in the nineteenth century.

\begin{tabular}{|l|l|}
\begin{tabular}{|l|l|}
\hline dëkk. Adduna ñépp dana ñu ko fekkisi. \\
Tallalal say loxo.
\end{tabular} & $\begin{array}{l}\text { be a vast no man's land where he will } \\
\text { establish a city. } \\
\text { find him there. }\end{array}{ }^{\text {i0 }}$ Hold out your hands.
\end{tabular}

\footnotetext{
9 Here, Taal refers to the holy city of Tuuba, which Bamba will found with his followers in 1887 ${ }^{10}$ This is a reference to the Murid holiday of the Grand Màggal, which draws millions of Murid devotees to Tuuba from throughout the world

This scene reflects the common belief that Umar Taal predicted the arrival of Amadu Bamba.
} 


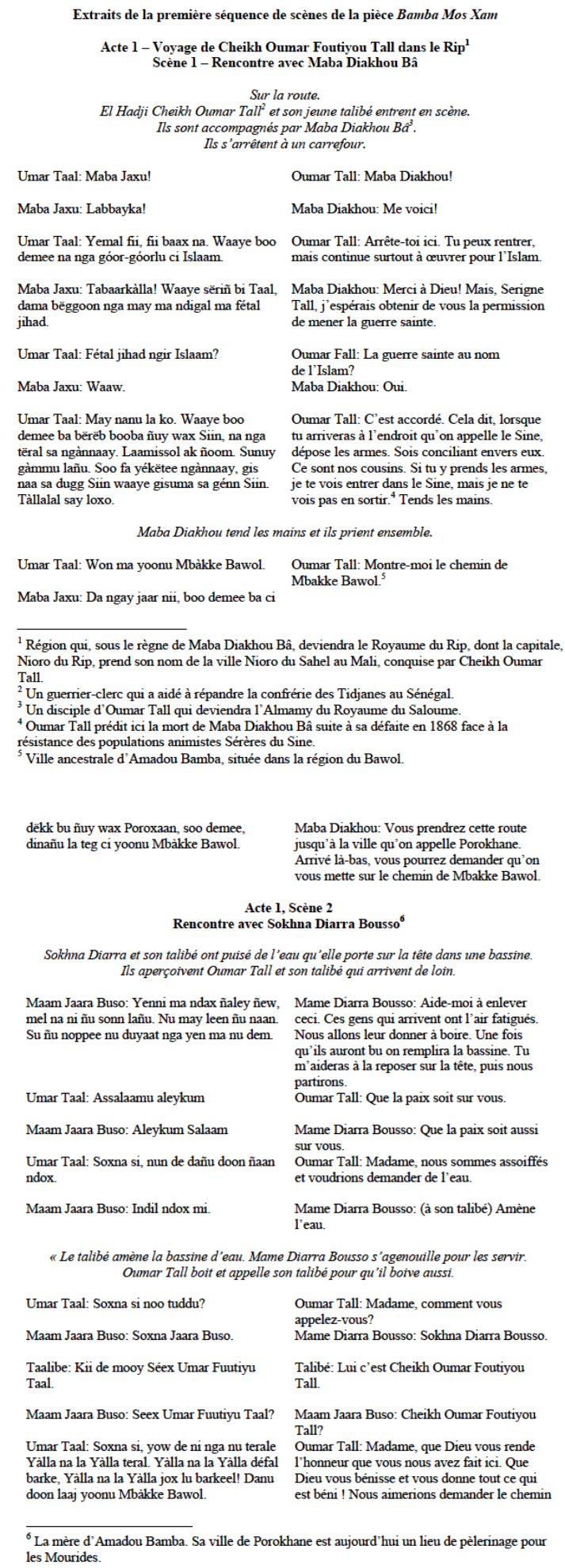




\begin{tabular}{|c|c|}
\hline $\begin{array}{l}\text { Maam Jaara Buso: Won leen yoonu Mbàkke } \\
\text { Bawol. }\end{array}$ & $\begin{array}{l}\text { de Mbakke Bawol. } \\
\text { Mame Diarra Bousso: (à son talibé) Indique- } \\
\text { leur le chemin de Mbakke Bawol. }\end{array}$ \\
\hline \multicolumn{2}{|c|}{ Le talibé de Sokhna Diarra Bousso leur montre le chemin de Mbakke Bawol. Ils s'en vont. } \\
\hline \multicolumn{2}{|c|}{$\begin{array}{c}\text { Acte 1, Scène } 3 \\
\text { Rencontre avec Mame Mor Anta Saly }\end{array}$} \\
\hline \multicolumn{2}{|c|}{$\begin{array}{l}\text { Oumar Tall arrive avec ses talibés à Mbakke Bawol. } \\
\text { Ils s'assoient sur la place publique. }\end{array}$} \\
\hline $\begin{array}{l}\text { Moor Anta Sali: Demal nga seetal ma gan } \\
\text { yale ca pénc ma. }\end{array}$ & $\begin{array}{l}\text { Mor Anta Saly (à son disciple): Va voir qui } \\
\text { sont ces êtrangers assis sur la place publique. }\end{array}$ \\
\hline \multicolumn{2}{|c|}{ Le disciple part pour les saluer. } \\
\hline Ndongo li: Assalaam aleykum. & $\begin{array}{l}\text { Disciple d'Anta Saly: Que la paix soit sur } \\
\text { vous. }\end{array}$ \\
\hline Umar Taal: Aleykum salaam. & Oumar Tall: Que la paix soit aussi sur vous. \\
\hline $\begin{array}{l}\text { Ndongo li: Man de, sëriñ Moor moo ma yónni } \\
\text { woon. Moo noon naa ko seetal ñi toog ci pénc } \\
\text { mi ñan lañu. }\end{array}$ & $\begin{array}{l}\text { Disciple: C'est Serigne Mor qui ménnoie } \\
\text { poour saluer cuxx qui se sont installés sur la } \\
\text { place publique et pour demander leurs noms. }\end{array}$ \\
\hline $\begin{array}{l}\text { Umar Taal: Boo demee nga ne ko Séex Omar } \\
\text { Fuutuyu Taal la. }\end{array}$ & $\begin{array}{l}\text { Oumar Tall: Va lui dire que c'est Cheikh } \\
\text { Oumar Foutiyou Tall. }\end{array}$ \\
\hline \multicolumn{2}{|c|}{$\begin{array}{l}\text { Le disciple s'en va pour le dire à Mame Mor Anta Saly, qui se déplace en personne pour } \\
\text { saluer leur hôte. Les denx hommes miment tes gestes d'une longue salutation suivie d'une } \\
\text { discussion chaleureuse. Ensuite : }\end{array}$} \\
\hline 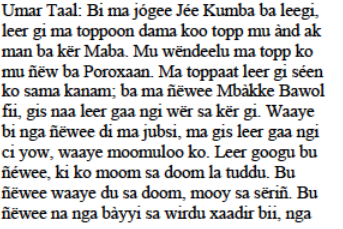 & $\begin{array}{l}\text { Oumar Tall: Depuis que j'ai quitté } \\
\text { Diégoumba, je suis une lumière qui m'a } \\
\text { amené jusqu'à chez Maba. Après, elle a } \\
\text { tournoyé et je l'ai suivie jusqu'à Porokhane. } \\
\text { Je l'ai suivie encore devant moi jusqu'a mon } \\
\text { arrivé icici à Mbakke Bawol. J'ai vu la lumière } \\
\text { tourner autour de ta maison. Lorsque tu es } \\
\text { venu vers moi, j'ai vu la lumière se poser sur } \\
\text { toi, mais elle ne t'appartient pas. Quand cette } \\
\text { lumeere arrivera on l'appellera ta progéniture. } \\
\text { Ce ne sera pas ton enfant, mais ton maitre. }\end{array}$ \\
\hline \multicolumn{2}{|l|}{ " Le père d'Amadou Bamba et un savant qadi. } \\
\hline 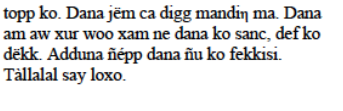 & $\begin{array}{l}\text { Lorsqu' il sera venu, quitte la voie xadiri }{ }^{8} \text { et } \\
\text { suis-le. Il ira jusque dans la savane où il } \\
\text { fondera, sur un enorme terrain vide, une } \\
\text { ville. } 2 \text { e monde entier ira l'y retrouver. }{ }^{10} \\
\text { Tends tes mains. }\end{array}$ \\
\hline & \\
\hline
\end{tabular}

\footnotetext{
${ }^{8}$ Anta Saly était un adepte de la Qadiriyya, une confrérie soufie fondée au douzième siècle, à Bagdad, et amenée au Sénégal au dix-huitième siècle

${ }^{9}$ Tall fait référence ici à la ville mouride de Touba, que Bamba fondera avec ses disciples en

${ }^{10}$ Une référence à la fête mouride du Grand Magal, qui attire à Touba des millions de Mourides de partout dans le monde.

'Cette scène reflète l'idée selon laquelle Oumar Tall aurait prédit l'arrivée d'Amadou Bamba
}

Oumar Tall aurait également cherché la ville de Touba avant sa découverte par Amadou Bamba

\section{NOTES}

1. Sur la politique et l'approche culturelles de Senghor, voir parmi d'autres : Cohen, Joshua, «Locating Senghor's École de Dakar: International and Transnational Dimensions to Senegalese 
Modern Art, c. 1959-1980», African Arts, vol. 51, n 3, 2018, 10-25 ; Diagne, Souleymane Bachir, Léopold Sédar Senghor: L'Art africain comme philosophie, Paris, Riveneuve éditions, 2007 ; Harney, Elizabeth, In Senghor's Shadow: Art, Politics, and the Avant-Garde in Senegal, 1960-1995, Durham, Duke University Press, 2004 ; Murphy, David (dir.), The First World Festival of Negro Arts, Dakar, 1966, Liverpool, Liverpool University Press, 2016 ; SNIPE, Tracey, Arts and Politics in Senegal, 1960-1996, Trenton NJ, Africa World Press, 1998. Sur l'art et la confrérie mouride, voir Camara, Sana, Sheikh Ahmadu Bamba: Selected Poems, Leiden, Brill, 2017 ; Dieng, Bassirou, L'Épopée de Cheikh Amadou Bamba de Serigne Moussa Kâ, Dakar, Presses Universitaires de Dakar, 2006 ; Ngom, Fallou, Muslims Beyond the Arab World: The Odyssey of Ajami and the Muridiyya, New York, Oxford University Press, 2016 ; Roberts, Allen F. et Mary Nooter Roberts, A Saint in the City: Sufi Arts of Urban Senegal, Los Angeles, UCLA Fowler Museum of Cultural History, 2003.

2. Cette recherche sur le théâtre populaire s'inscrit dans le cadre d'un ouvrage en cours sur les différents usages et adaptations de la scène théâtrale au Sénégal. Voir également l'article "Crafting the Popular Stage Space in Urban Senegal », qui sera publié en 2020 dans Research in African Literatures.

3. Kàddu Yaraax, parmi les compagnies de théâtre populaire les plus en vue du Sénégal, organise depuis 2005 un festival international de théâtre forum dans la municipalité de Yarakh.

4. Baba Ndiaye, intervention, "Théâtre, diversité culturelle et développement durable» (conférence du FEST'RAIL), le 14 mai 2016 à Thiès.

5. Ces interlocuteurs sont: Cheikh Fall (ancien directeur de la troupe); Selbe Diagne et Abdoulaye Faye (anciens artistes interprètes de la troupe) ; Mor Sadio Niang (ancien directeur du centre culturel de Kaolack); Oumar Thiam (percussionniste de renommée internationale, originaire de Kaolack); Yelimane Fall (artiste peintre de renommée internationale et figure culturelle importante du milieu du mouridisme); Fallou Fall Mbaor (petit-fils de Mame Cheikh Ibrahima Fall, et figure médiatique de la confrérie mouride) ; Cheikh Samb (petit-fils de l'acteur principal de Bamba Mos Xam et journaliste dans les médias mourides de Touba); Beugue Fallou (ancien diwaankaat, ou conteur professionnel, de Touba). Nous avons bénéficié dans nos recherches de l'assistance d'Ousmane Guèye de Touba et Pikine.

6. Canella, Gino et Brian Valente-Quinn (réals.), Bamba, The Taste of Knowledge, film documentaire, 2018, 22 mins, https://vimeo.com/264755999.

7. Féral, Josette, «Introduction: Towards a Genetic Study of Performance - Take 2 », Theatre Research International, vol. 33, n 3, 2008, 223-333 ; Léger, Nathalie et Almuth Grésillon, « Scènes de genèses théâtrales ", Genesis, nº 26, 2005, p. 19.

8. Siaud, Florent, "La génétique de la mise en scène à l'épreuve de l'expérience humaine ", $L a$ fabrique du texte à l'épreuve de la génétique, Olga Anokhina et Fatiha Idmhand (dirs.), Éditions des archives contemporaines, France, 2018, p. 32.

9. Cole, Catherine, Ghana's Concert Party Theatre, Bloomington, Indiana University Press, 2001, p. 2. 10. Pour citer quelques exemples : Cole, op. cit. ; Donkor, David, Spiders of the Market: Ghanaian Trickster Performance in a Web of Neoliberalism, Bloomington, Indiana Unversity Press, 2016; Edmondson, Laura, Performance and Politics in Tanzania: The Nation on Stage, Bloomington, Indiana University Press, 2007 ; Neveu-Kringelbach, Hélène, Dance Circles: Movement, Morality and SelfFashioning in Urban Senegal, Oxford, Berghahn, 2013 ; Shipley, Jesse Weaver, Trickster Theater: The Poetics of Freedom in Urban Africa, Bloomington, Indiana University Press, 2015.

11. Cette situation a suscité de vives critiques dénonçant l'écart entre la floraison d'études sur la littérature ou le théâtre africains de langue française et la quasi-absence de travaux sur la production littéraire ou théâtrale dans et pour les pays concernés. Voir : Conteh-Morgan, John, Theatre and Drama in Francophone Africa: A Critical Introduction, Cambridge, Cambridge University Press, 1994, 221 ; Le Lay, Maëline, La parole construit le pays. Théâtre, langues et didactisme au Katanga (République Démocratique du Congo), Paris, Honoré Champion, 2014, p. 11. 
12. TAYLOR, Diana, The Archive and the Repertoire: Performing Cultural Memory in the Americas, Durham, Duke University Press, 2003, p. 16-21. Dans son étude Taylor valorise l'apport de ce qu'elle nomme le "répertoire ", défini comme l'ensemble de savoirs et de pratiques qui se transmettent par des moyens corporels, nécessitent une présence participative, et s'avèrent donc de nature instable et éphémère à la différence des principes de toute archive. Taylor souligne que le répertoire, bien qu'éphémère, préserve et transforme à la fois des chorégraphies « de sens » et nous permet ainsi de retracer les traditions et les influences.

13. Cheikh Fall, entretiens des 15 et 21 mai 2016 à Kaolack. Pour la retranscription de cette pièce de langue wolof, nous avons bénéficié de l'assistance d'Abdoulaye Karel Dieng, directeur du Salon africain des langues et cultures (SALC) et de Paap Alsaan Sow, linguiste et traducteur.

14. Le ndigal est souvent discuté dans les cadres politique et électoral du pays. Le terme peut signifier l'acte, de la part d'un marabout, de donner une consigne de vote à sa communauté de talibés. Ici le terme signifie plutôt la permission ou l'autorisation de la part d'une autorité religieuse.

15. Le comédien Selbe Diagne, ancien membre de la troupe, a confirmé la nécessité de se montrer en artiste «total », capable de jouer, danser, chanter et interpréter plusieurs rôles, comme il l'a fait lui-même durant ses longues années avec la troupe. Entretien le 25 décembre 2016 à Kaolack. 16. Fallou Fall Mbaor a évoqué les foules d'enfants qui s'amassaient sur les toits des immeubles (entretien le 17 décembre 2016 à Touba); Fallou Ngom, spécialiste de la littérature ajami et spectateur de la pièce dans sa région natale de Casamance, s'est souvenu, lors d'une conférence, des groupes d'enfants qui, en guise de paiement, donnaient des seaux remplis d'eau aux comédiens assoiffés (intervention à l'université de Boston, le 25 mars 2019).

17. Balme, Christopher, Decolonizing the Stage: Theatrical Syncretism and Post-Colonial Drama, Oxford, Clarendon Press, 1999.

18. Jézéquel, Jean-Hervé, «Le "théâtre des instituteurs" en AOF (1930-1950) : pratique socioculturelle et vecteur de cristallisation de nouvelles identités urbaines ", Fêtes urbaines en Afrique: espaces, identités et pouvoirs, Odile Georg (dir.), Paris, Karthala, 1994, p. 181-200.

19. Roberts, op. cit.

20. Yelimane Fall affirme que, pour les mourides ainsi que pour les autorités de Touba, le respect et l'exactitude des faits établis par le dogme de la confrérie constituent un enjeu majeur dans toute demande d'autorisation de représenter un quelconque aspect de la vie ou des enseignements de Bamba (entretien le 18 mai 2016 à Pikine).

21. C'est la formule qu'emploie Fallou Ngom dans son étude de la littérature wolofal (op. cit.). Sur cette littérature, voir aussi : Camara, Sana, «A'jami Literature in Senegal: The Example of Sëriñ Muusaa Ka, Poet and Biographer ", Research in African Literatures, vol. 28, n 3, 1997, p. 163-182 ; Ngom, op. cit.

22. Le poème est publié dans son intégralité dans une version bilingue wolof-français dans DIENG, op. cit.

23. Ngom, op. cit., p. 10.

24. Cheikh Samb, journaliste impliqué dans les médias mourides, a également insisté sur le rôle primordial que jouaient les écrits de Moussa Kâ et son importance pour la pièce Bamba Mos Xam (entretien le 17 décembre à Touba). La retranscription du texte dirigé par Cheikh Fall comporte en effet des références au texte de Kâ.

25. Yelimane Fall nous explique que l'avènement de la tournée coïncide avec un accroissement notable du nombre d'adeptes, attribuable en partie, à son avis, au phénomène de Bamba Mos Xam. Fall attribue son propre choix de devenir mouride à l'effet d'une représentation du spectacle à Saint-Louis, sa ville natale.

26. Cheikh Samb, petit-fils de Demba Ndiaye, confirme le statut qu'avait son grand-père au sein de la communauté mouride en raison de son rôle dans Bamba Mos Xam (entretien le 17 décembre 2016 à Touba). Soukaye Ndiaye, marchande de rue à Touba, nous a longuement parlé de l'effet 
que faisait ce comédien qui imitait à merveille le cheikh (entretien le 17 décembre 2016 à Touba). Cheikh Fall a également insisté sur l'érudition de Demba Ndiaye (entretien le 25 décembre à Kaolack).

27. Retranscription de la pièce Bamba Mos Xam, p. 4.

28. Cruise O’Brien, Donald, «The Senegalese Exception » Africa, vol. 66, n 3, 1996, p. 458-464.

29. Yelimane Fall, spectateur de la pièce en plusieurs villes et contextes, affirme avoir souvent vu des spectateurs monter sur scène pour intervenir physiquement dans l'action (entretien le 15 décembre à Guédiawaye).

30. Retranscription de la pièce Bamba Mos Xam, p. 21-23.

31. Babou, Cheikh, Fighting the Great Jihad: Amadu Bamba and the Founding of the Muridiyya of Senegal, 1853-1913, Athens, Ohio University Press, 2007, p. 93.

32. Dans le cadre d'un discours publié sur le site du théâtre, Massamba Guèye, ancien directeur de Sorano, parle brièvement d'une représentation du spectacle au Théâtre national : (http:// www.compagnietheatresorano.com/rubriques.php?rub=article.php\&id_article=62).

33. Benga, Ndiouga, "Mise en scène de la culture et espace public au Sénégal ", Afrique et Développement, vol. 35, n 4, 2010, p. 237-260.

34. Voir: Hill, Joseph, "Baay is the Spiritual Leader of the Rappers": Performing Islamic Reasoning in Senegalese Hip-hop ", Contemporary Islam, vol. 10, n 2, 2016, p. 267-287 ; Mclaughlin, Fiona, "Islam and Popular Music in Senegal: The Emergence of a "New Tradition" ", Africa: Journal of the International African Institute, vol.67, $\mathrm{n}^{\circ} 4$, 1997, p.560-581; Niang, Abdoulaye, " "Preaching Music" and Islam in Senegal: Can the secular mediate the religious? The case of rap and mbalax music ", African Communication Research, vol. 2, n 1, 2009, p. 61-84 ; Roberts, Allen F., "Citoyennetés visuelles en compétition dans le Sénégal contemporain », Les Arts de la citoyenneté au Sénégal, Mamadou Diouf et Rosalind Fredericks (dir.), Paris, Karthala, 2013, p. 195-236.

35. Adama Guèye, entretien le 15 décembre 2016 à Thiaroye.

36. Ces rencontres ont lieu les 22 et 23 décembre 2016 à Dakar, et se poursuivent les 24 et 25 décembre à Kaolack. Les discussions font l'objet d'une partie du film documentaire, Bamba, the Taste of Knowledge.

37. Représentation du 23 décembre en présence de Cheikh Fall au centre culturel de Yeumbeul.

38. Voir Tang, Patricia, Masters of the Sabar: Wolof Griot Percussionists of Senegal, Philadephia, Temple University Press, 2007, p. 103.

\section{RÉSUMÉS}

Cet article retrace l'histoire d'une création théâtrale ambulante d'inspiration religieuse qui, au cours d'une tournée continue de plus de 15 ans entamée vers la fin des années 1960, a marqué profondément l'imaginaire national et religieux du Sénégal. Le spectacle en langue wolof, intitulé Bamba Mos Xam, racontait la vie et l'œuvre de Cheikh Amadou Bamba, saint fondateur soufi de la confrérie sénégalaise des mourides. Par son choix de matière, il a également pu renouveler de manière radicale la nature et les usages possibles de l'espace scénique dans ce pays. Ici, un travail de reconstitution nous permettra de nous interroger sur l'approche théâtrale de cette création et les raisons de son quasi-oubli de la part des institutions culturelles, religieuses et académiques.

This article retraces the history of a religiously inspired traveling theatrical performance that began in the late 1960s and, through the course of a continuous tour of over 15 years, left a 
profound mark on the national and religious cultural heritage of Senegal. The Wolof-language performance, entitled Bamba Mos Xam, retold the life and work of Shaykh Amadu Bamba, founding Sufi saint of the Senegalese brotherhood of the Murids. In its choice of subject material, it also radically innovated the nature and possible uses of the theatrical stage space in this country. Through a reconstitution of the play, this project reflects on the theatrical approach of Bamba Mos Xam as well as the reasons for its near omission from cultural, religious and academic forms of institutional memory.

\section{INDEX}

Mots-clés : théâtre sénégalais, art mouride, théâtre populaire, culture sénégalaise Keywords : senegalese theatre, murid art, popular theatre, senegalese culture

\section{AUTEUR}

\section{BRIAN VALENTE-QUINN}

BRIAN VALENTE-QUINN est professeur adjoint à l'Université du Colorado à Boulder où il enseigne la littérature africaine de langue française. Ses recherches portent sur le théâtre africain francophone, et il prépare actuellement un livre sur les usages et innovations du jeu scénique au Sénégal. 